\title{
AMPK-SIRT1 pathway dysfunction contributes to neuron apoptosis and cognitive impairment induced by sevoflurane
}

\author{
LIWEI LIU ${ }^{1}$, CHAO LIU ${ }^{2}$ and LIN FANG ${ }^{3}$ \\ ${ }^{1}$ Department of Anesthesiology, Shanghai Tenth People's Hospital, Tongji University School of Medicine, \\ Shanghai 200072; ${ }^{2}$ Institute of Cardiovascular Diseases, Tianjin Chest Hospital, Tianjin 300457; \\ ${ }^{3}$ Department of Thyroid and Breast Surgery, Shanghai Tenth People's Hospital, \\ Tongji University School of Medicine, Shanghai 200072, P.R. China
}

Received December 30, 2019; Accepted June 5, 2020

DOI: $10.3892 / \mathrm{mmr} .2020 .11694$

\begin{abstract}
The anesthetic sevoflurane (Sev) is widely used because of its low blood-gas partition coefficient and lack of pungency. However, the application of Sevmay lead to cognitive impairment later in life. Previous results have indicated that exposure to Sev-induced neuronal apoptosis and cognitive dysfunction in a rat model, but much work remains to elucidate the mechanism. In the present study, inhibition in the AMP-activated protein kinase/Sirtuin 1 (AMPK/SIRT1) signaling pathway and a decrease in AMPK/SIRT1 activity was found to occur concomitantly in neuronal apoptosis induced by Sev. AICAR, an activator of AMPK, was able to suppress Sev-induced neuronal apoptosis and SIRT1 activity reduction in vitro. Further animal studies also showed that AICAR treatment blocked the deleterious cognition and AMPK/SIRT1 activity reduction in the cognition impairment rats induced by Sev. Taken together, it was concluded that the AMPK/SIRT1 signaling pathway mediates neuronal apoptosis and cognition impairment induced by Sev. The study provides evidence that AMPK activation ameliorates Sev-induced cognitive deficits.
\end{abstract}

\section{Introduction}

Sevoflurane (Sev) is widely used in pediatric anesthesia. Unfortunately, it has been demonstrated that Sev has potential neurotoxic effects on the developing brain and cognition, even in adolescence $(1,2)$. Sev treatment can harm neural stem cells, neuronal, lung and cancer cells, Substantial evidence has accumulated to support the conclusion that Sev leads to neuronal

Correspondence to: Professor Lin Fang, Department of Thyroid and Breast Surgery, Shanghai Tenth People's Hospital, Tongji University School of Medicine, 301 Yanchang Middle Road, Jingan, Shanghai 200072, P.R. China

E-mail: fanglin1228@163.com

Key words: sevoflurane, apoptosis, neuron, AMPK/SIRT1, cognition apoptosis $(3,4)$. The present study sought to elucidate this important mechanism of neuronal apoptosis induced by Sev.

AMP-activated protein kinase (AMPK) is a key regulator to maintain the stability of energy metabolism by modulating various regulatory signaling pathways at the cellular level. It consists of a catalytic subunit $(\alpha)$ and two regulatory subunits $(\beta$ and $\gamma$ ), which are activated by the phosphorylation of threonine 172 (Thr172) (5,6). AMPK downregulation occurs in animal models with metabolic syndromes (7). Sirtuin 1 (SIRT1), a NAD ${ }^{+}$dependent protein deacetylase, has an important role in the regulation of physiological and pathological processes such as apoptosis, metabolism and differentiation, through the deacetylation of intracellular signaling factors $(8,9)$. AMPK and SIRT1 have similar roles in cellular metabolism and survival (10). Past work has revealed that phosphorylated-(p-)AMPK can activate SIRT1, and the activated SIRT1 regulates the formation of the autophagic vacuole (11-13). There is a positive feedback loop interaction between AMPK and SIRT1 (14). It has also been shown that AMPK and SIRT1 are associated with cell aging and neurological disorders $(15,16)$. Shah et al $(17)$ revealed that the AMPK/SIRT1 pathway is involved in the modulation of $\mathrm{A} \beta$ deposition and cognitive functions in Alzheimer's disease rats. Yet, the contribution of AMPK/SIRT1 in neuronal apoptosis and cognitive impairment induced by Sev exposure is still not clear.

In the present study, the role of the AMPK/SIRT1 pathway in Sev-induced neuronal apoptosis and cognition impairment was examined in rats. The study may offer the possibility to address the question of whether AMPK could be a new drug target for the prevention of cognitive impairment induced by Sev.

\section{Materials and methods}

Materials. Sev, AICAR, Annexin V-FITC Apoptosis Detection Kit and Hoechst 33342 were purchased from Sigma-Aldrich (Merck KGaA). Fetal bovine serum (FBS) was purchased from Gibco (Thermo Fisher Scientific, Inc.). The following primary antibodies were purchased from Abcam: Anti-actin (cat. no. ab179467), anti-total-(t-) AMPK (cat. no. ab80039), anti-p-AMPK (cat. no. ab133448), 
anti-SIRT1 (cat. no. ab189494). AMPK (cat. no. bf30053) and SIRT1 ELISA kits (cat. no. bf90658) were purchased from Northern Institute of Biotechnology of China.

Cell culture and Sev exposure. PC12 cells, originally derived from a pheochromocytoma of rat adrenal medulla, were used to evaluate the involved mechanism of Sev-induced cell apoptosis. PC12 cells were obtained from Shanghai Institute for Biological Science Cell Bank, CAS. The cells were cultured in DMEM with 5\% FBS in an incubator at $37^{\circ} \mathrm{C}$ with $5 \% \mathrm{CO}_{2}$. For Sev exposure, the cell culture plates were placed in the airtight incubator connected to an anesthesia machine that was used to supply Sev into the incubator. PC12 cells were exposed to $3 \% \mathrm{Sev}$ for $24 \mathrm{~h}$ (18). For the intervention studies, AICAR, an AMPK activator, was used to treat $\mathrm{PC} 12$ cells at a concentration of $0.5 \mathrm{mM}$ for $1 \mathrm{~h}$ at $37^{\circ} \mathrm{C}$ prior to $\mathrm{Sev}$ treatment.

Annexin V-FITC/PI staining. The cell apoptosis was evaluated by flow cytometry analysis using Annexin V-FITC/PI apoptosis analysis, according to the manufacturer's instructions (Shanghai Yeasen Biotechnology Co., Ltd.). Briefly, PC12 cells were exposed to Sev for $24 \mathrm{~h}$, and harvested using trypsin. The harvested cells were resuspended in $1 \mathrm{X}$ binding buffer (Shanghai Yeasen Biotechnology Co., Ltd.) at a concentration of $1 \times 10^{6} \mathrm{cells} / \mathrm{ml}$. A total of $5 \mu \mathrm{l}$ of Annexin V-FITC and $10 \mu \mathrm{l}$ PI staining solution was subsequently added to the suspensions. The suspensions were vortexed gently, and $400 \mu \mathrm{l}$ of $1 \mathrm{X}$ binding buffer was added and incubated for $5 \mathrm{~min}$ at room temperature in the dark. The fluorescence of FITC and PI was analyzed by flow cytometry. Both early apoptotic (Annexin $\mathrm{V}-\mathrm{FITC}^{+} / \mathrm{PI}^{-}$) and late apoptotic (Annexin V-FITC ${ }^{+} / \mathrm{PI}^{+}$) cells were included in cell apoptosis determinations.

Hoechst 33342 staining. A total of $6 \times 10^{5} \mathrm{PC} 12$ cells were seeded into 6-well plates and exposed to $\mathrm{Sev}$ at $37^{\circ} \mathrm{C}$ for $24 \mathrm{~h}$. A total of $1 \mathrm{ml}$ of $4 \%$ paraformaldehyde was added to each well for $10 \mathrm{~min}$ for fixation and washed twice with PBS. A total of $10 \mathrm{mg} / \mathrm{l}$ Hoechst 33342 was added and stained for $10 \mathrm{~min}$ at room temperature. Cell apoptosis was observed under a fluorescence microscope. Apoptotic cells were characterized by rounded cell morphology, half-moon nuclei with condensed chromatin. The apoptotic cells were counted the average no. of cells $/ \mathrm{mm}^{2}$ in 10 fields.

Western blotting. After incubation with Sev for $24 \mathrm{~h}, \mathrm{PC} 12$ cells were collected, rinsed twice with cold PBS and lysed by RIPA buffer (Thermo Fisher Scientific, Inc.) containing $1 \mathrm{mM}$ PMSF on ice for $30 \mathrm{~min}$, followed by centrifugation at $12,000 \mathrm{x} \mathrm{g}$ for $5 \mathrm{~min}$ at $4^{\circ} \mathrm{C}$. A total of $40 \mu \mathrm{g}$ of total protein was loaded in each well and resolved by $10 \%$ SDS-PAGE. After electrophoresis, samples were transferred onto polyvinylidene fluoride (PVDF) membranes, which were subsequently blocked with $5 \%$ skimmed milk for $1 \mathrm{~h}$ and incubated with anti-t-AMPK (1:800), anti-p-AMPK (1:500), anti-SIRT1 $(1: 1,000)$ and anti-actin $(1: 2,000)$ primary antibodies at $4^{\circ} \mathrm{C}$ for $12 \mathrm{~h}$. The membrane was washed three times with PBST (0.1\% Tween-20), and then incubated with the second antibody (goat anti-rabbit 1:2,000 in blocking solution) at $37^{\circ} \mathrm{C}$ for $2 \mathrm{~h}$. The membrane was then washed three times with PBST. Then, the signals were visualized with chemiluminescence reagents (Thermo Fisher Scientific, Inc.). Results were semi-quantified via Quantity One software (version 4.4; Bio-Rad Laboratories, Inc.).

Measurement of AMPK and SIRT1 levels. AMPK and SIRT1 levels in cell culture medium and hippocampus homogenate were measured by commercially available ELISA kits.

Animals and Sev exposure. A total of 20 male Sprague-Dawley (SD) rats ( $28 \pm 2$ days old; weight $100 \pm 10 \mathrm{~g}$ ) were used. Animals were housed with free access to food and water at the temperature of $22 \pm 2^{\circ} \mathrm{C}$, humidity of $55 \pm 5 \%$ and a 12 -h light/dark cycle. The rats were randomly assigned to the control (Con) and Sev groups. The Con group was not exposed to Sev. Sev group received inhalation of 3\% Sevin $\mathrm{O}_{2} / \mathrm{N}_{2}$ (fraction of inspired oxygen $50 \%$ ) for $2 \mathrm{~h}$ daily for 5 days consecutively. The dose of $\mathrm{Sev}$ was determined as previously described (19). For the intervention studies, AICAR, an AMPK activator, was administered to the rats via an intraperitoneal injection for $2 \mathrm{~h}$ at a dose of $500 \mathrm{mg} / \mathrm{kg}$ prior to Sev treatment. The health and behavior of animals were monitored every day. The present study was performed in strict accordance with the recommendations in the Guide for the Care and Use of Laboratory Animals of the National Institutes of Health. All efforts to improve animal welfare were taken, including to minimize suffering and distress, use of an aesthetics or special housing conditions.

Water maze. Rats were trained in a $1.5 \mathrm{~m}$ diameter open-field water maze filled with water $\left(26^{\circ} \mathrm{C}\right)$ and made opaque with latex liquid. Prominent extra-maze visual cues around the room remained in fixed positions throughout the experiment. During behavioral testing, animals were required to locate a hidden submerged platform $10 \mathrm{~cm}$ in diameter $(1.5 \mathrm{~cm}$ below the surface), which remained in the same position across trials for individual animals but was counterbalanced across animals. The animals were given four trials per day for 4 days. Trials began as the rat was placed in the pool facing the side wall at a start position and ended once the animal had found the platform; if the rat had not found the platform within $120 \mathrm{sec}$, it was guided there by hand. A video camera mounted to the ceiling directly above the center of the maze was used in conjunction with the animal tracking system. Test animals underwent Sev exposure for 5 days followed by the water maze experiment for 5 days. On day 11, the animals were sacrificed to explore further mechanisms under anesthesia with mebumal sodium $(50 \mathrm{mg} / \mathrm{kg})$ by intraperitoneal injection.

Hippocampus homogenate. After the last water maze was completed, the rats were anesthetized and euthanized by mebumal sodium. The hippocampus was removed and the rats were verified dead. Then, the hippocampus was weighed, transferred into a glass homogenizer and five volumes of precooled PBS (0.02 mol/l, pH 7.0-7.2) was added. The tissue was fully ground and then treated with ultrasonic crushing. After centrifugation of the prepared homogenate at $10,000 \mathrm{xg}$ for $5 \mathrm{~min}$ at $4^{\circ} \mathrm{C}$, the hippocampal homogenate was found in the supernatant.

Statistical analyses.t-tests and two-way ANOVA, followed by a Bonferroni post hoc analysis, were performed for comparisons 
A

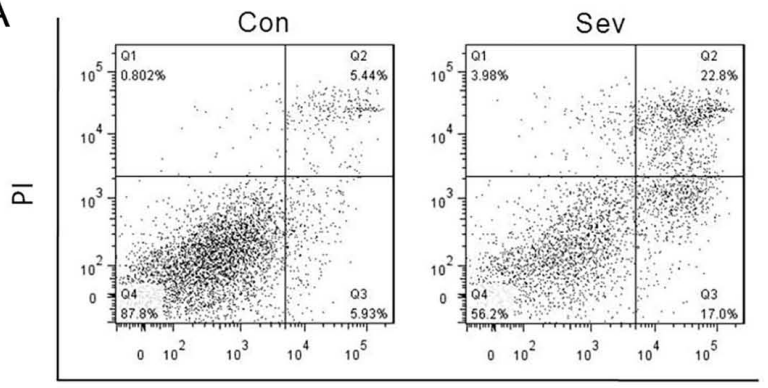

Annexin V
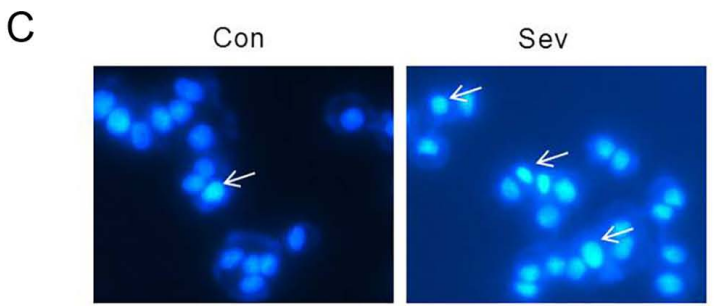

B

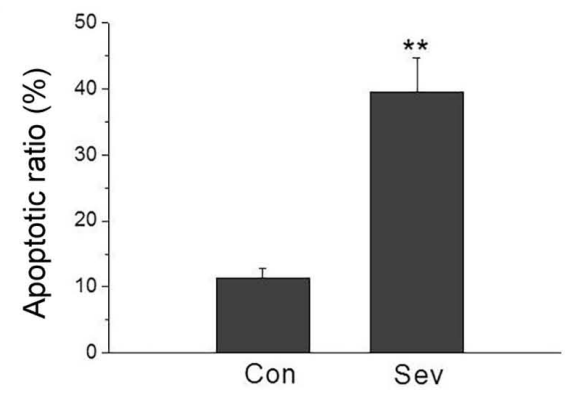

D

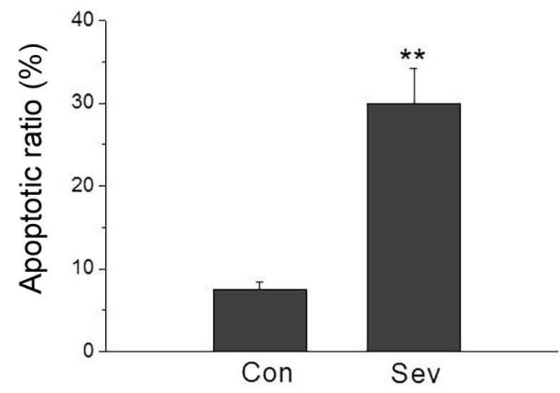

Figure 1. Sev induces neuronal apoptosis. (A and B) Sev induced cell apoptosis by Annexin V-FITC assay. The lower left population of cells in each plot represents viable cells which excluded PI and did notbind Annexin V, while the upper left population cells comprise necrotic cells, which did not exclude PI and were not stained with FITC-labeled Annexin V. The lower and upper right populations correspond to early apoptotic and late apoptotic cells, respectively. The percentages of early and late apoptotic cells, non-apoptotic and necrotic cells are shown in the right lower quadrant, right upper quadrant, left lower quadrant and left upper quadrant, respectively. (C and D) Sev induced cell apoptosis by Hoechst 33342 staining. Apoptotic cells were characterized by rounded cell morphology, half-moon nuclei with condensed chromatin.Arrowsindicate apoptotic cells. ${ }^{* *} \mathrm{P}<0.01$ vs. Con. $\mathrm{n}=5$. Sev, sevoflurane; Con, control.

between groups. Statistical analysis was conducted on a standard software package (SAS ${ }^{\circledR}$ version 10.0; SAS Institute, Inc.) was used. All data are presented as the mean \pm SEM. Each experiment was repeated $>4$ times. $\mathrm{P}<0.05$ was considered to indicate a statistically significant difference.

\section{Results}

Sev induces neuronal apoptosis. Cell apoptosis was assessed by Hoechst 33342 staining and Annexin V-FITC/PI staining. To investigate the effects of Sev on neuronal apoptosis, the percentage of apoptotic cells was measured using Annexin V/PI staining. The results showed that the percentage of apoptotic cells was significantly increased after treatment with Sev (Fig. 1A and B). Furthermore, Hoechst 33342 staining also showed that Sev caused neuronal apoptosis (Fig. 1C and D).

Inhibition of AMPK/SIRT1 signaling is associated with Sev-mediated neuronal apoptosis. The AMPK and SIRT1 protein expression levels were assessed by western blot analysis. As shown in Fig. 2A and B, p-AMPK and SIRT1 protein expression levels were significantly decreased after Sev treatment. The t-AMPK protein expression had no significant difference between the two treatment groups. The ratio of $\mathrm{p}-\mathrm{AMPK} / \mathrm{t}-\mathrm{AMPK}$ decreased in the group treated with Sev. Next, AMPK and SIRT1 activity was examined in cell culture medium by ELISA. There was a significant inhibitory effect of Sev on AMPK and SIRT1 activity (Fig. 2C and D). These results indicated that inhibition of AMPK/SIRT1 signaling was related to neuronal apoptosis in response to $\mathrm{Sev}$.
AMPK activation blocks neuronal apoptosis and the decline in SIRT1 activity induced by Sev. To examine the role of AMPK/SIRT1 signaling in Sev-induced neuronal apoptosis, the neurons were treated with the AMPK activator-AICAR at a concentration of $0.5 \mathrm{mM}$, and the percentage of apoptotic cells was evaluated using Annexin V/PI staining. As shown in Fig. 3A and B, AICAR was able to attenuate the neuronal apoptosis induced by Sev. AICAR also blocked the decline inSIRT1 activity induced by Sev (Fig. 3C). These results suggested that neuronal apoptosis caused by Sev was dependent on the AMPK/SIRT1 signaling pathway.

AMPK activation improves cognition and restores in vivo SIRT1 levels decreased by Sev. To examine the role of AMPK/SIRT1 signaling in Sev-induced cognition impairment, the rats were treated with the AMPK activator-AICAR at $500 \mathrm{mg} / \mathrm{kg}$, and $\operatorname{cog}$ nition was evaluated using the water maze test. As shown in Fig. 4A-C, Sev increased the escape latency time and distance in the water maze. The escape latency time and distance were notably decreased in the AICAR-treated rats. Furthermore, Sev decreased the AMPK and SIRT1 activity in the hippocampus. When AICAR was administered to rats, the AMPK and SIRT1 activity in the hippocampus was significantly increased (Fig. 4D). These results suggested that AMPK activation could improve the cognitive impairment caused by Sev.

\section{Discussion}

Sev is delivered via the lungs as a volume percent of inspired gas. Sev has been described as the agent of choice for 
A

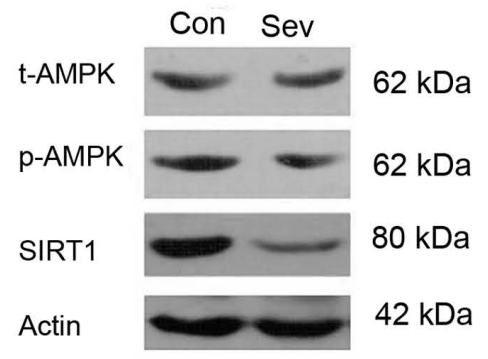

C

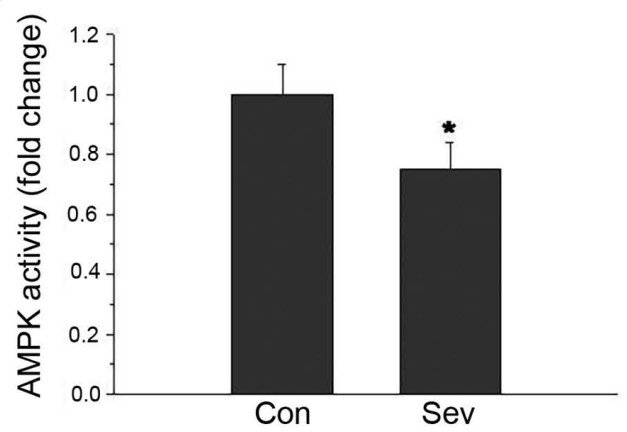

$\mathrm{B}$

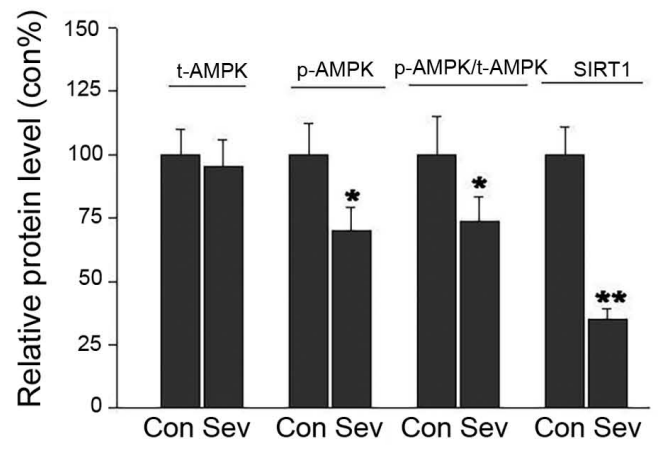

D

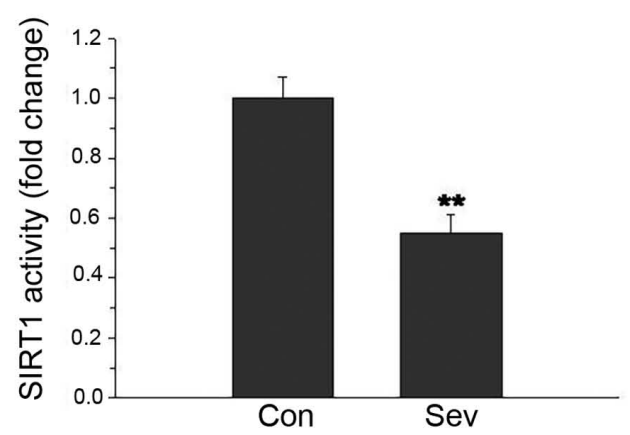

Figure 2. Inhibition of AMPK/SIRT1 inhibition is related to the neuronal apoptosis induced by Sev. (A) Representative western blots of t-AMPK, p-AMPK and SIRT1 expression levels in neurons treated with Sev. (B) The expression levels of p-AMPK, SIRT1 and the ratio of p-AMPK/t-AMPK were decreased in the group treated with Sev. (C and D) The AMPK and SIRT1 activities in the Sev group were decreased in the media of cell culturesas detected by ELISA. "P<0.05, ${ }^{*} \mathrm{P}<0.01$ vs. Con. $\mathrm{n}=5$. Sev, sevoflurane; Con, control; p-, phosphorylated; t-, total; AMPK, AMP-activated protein kinase; SIRT1, Sirtuin 1.

A

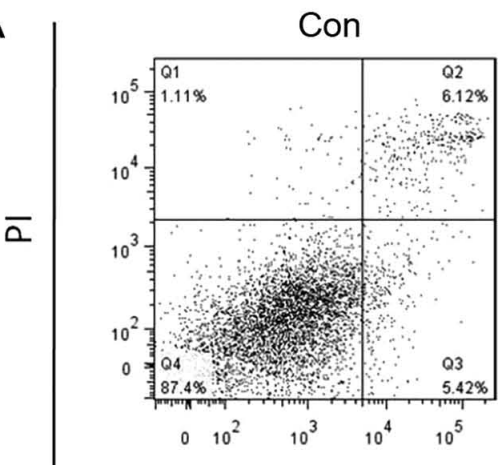

Sev

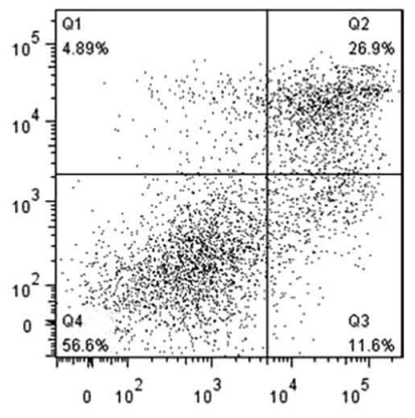

AICAR+sev

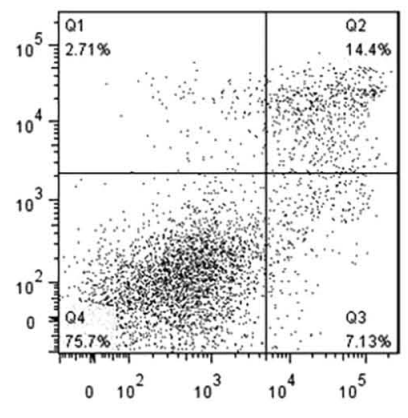

Annexin $\mathrm{V}$

B

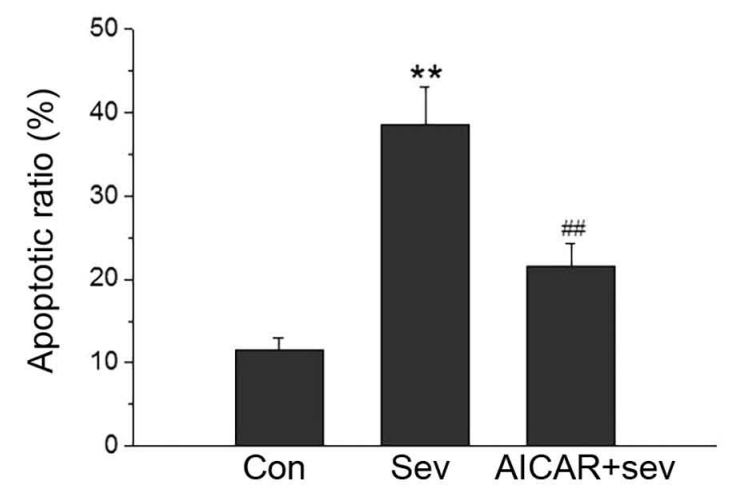

C

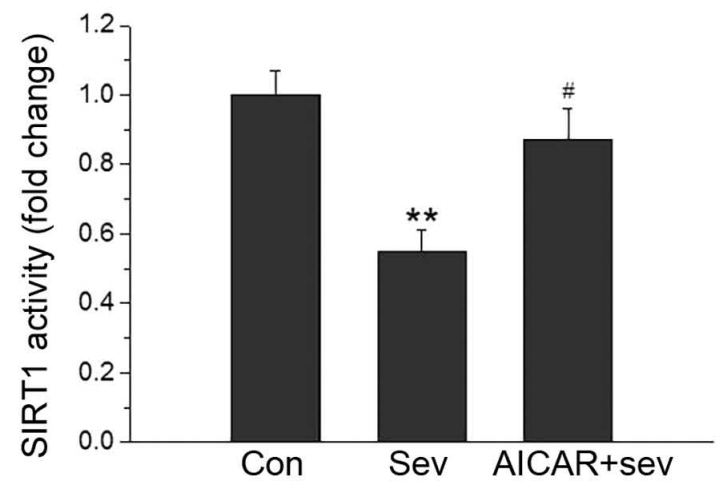

Figure 3. AICAR reduces the percentage of apoptotic cells and increases SIRT1 activity in Sev-induced neuronal apoptosis. (A and B) AICAR restrained the cell apoptosis after Sev treatment by Annexin V-FITC assay. The percentages of early and late apoptotic cells, non-apoptotic and necrotic cells are shown in the right lower quadrant, right upper quadrant, left lower quadrant and left upper quadrant, respectively. (C) AICAR increased the SIRT1 activity after Sev treatment as detected by ELISA. ${ }^{* *} \mathrm{P}<0.01$ vs. Con; ${ }^{\#} \mathrm{P}<0.05,{ }^{\# \#} \mathrm{P}<0.01$ vs. Sev. $\mathrm{n}=4$. Sev, sevoflurane; Con, control; SIRT1, Sirtuin 1. 
A

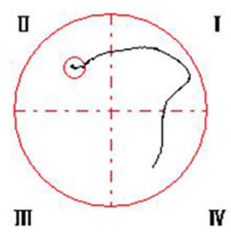

Con
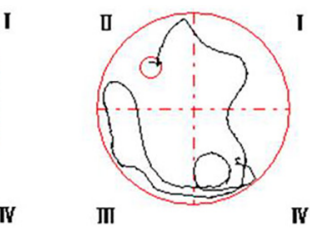

Sev

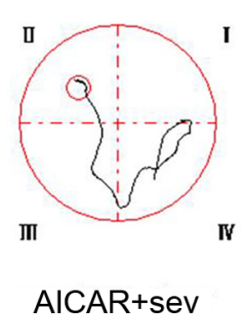

B
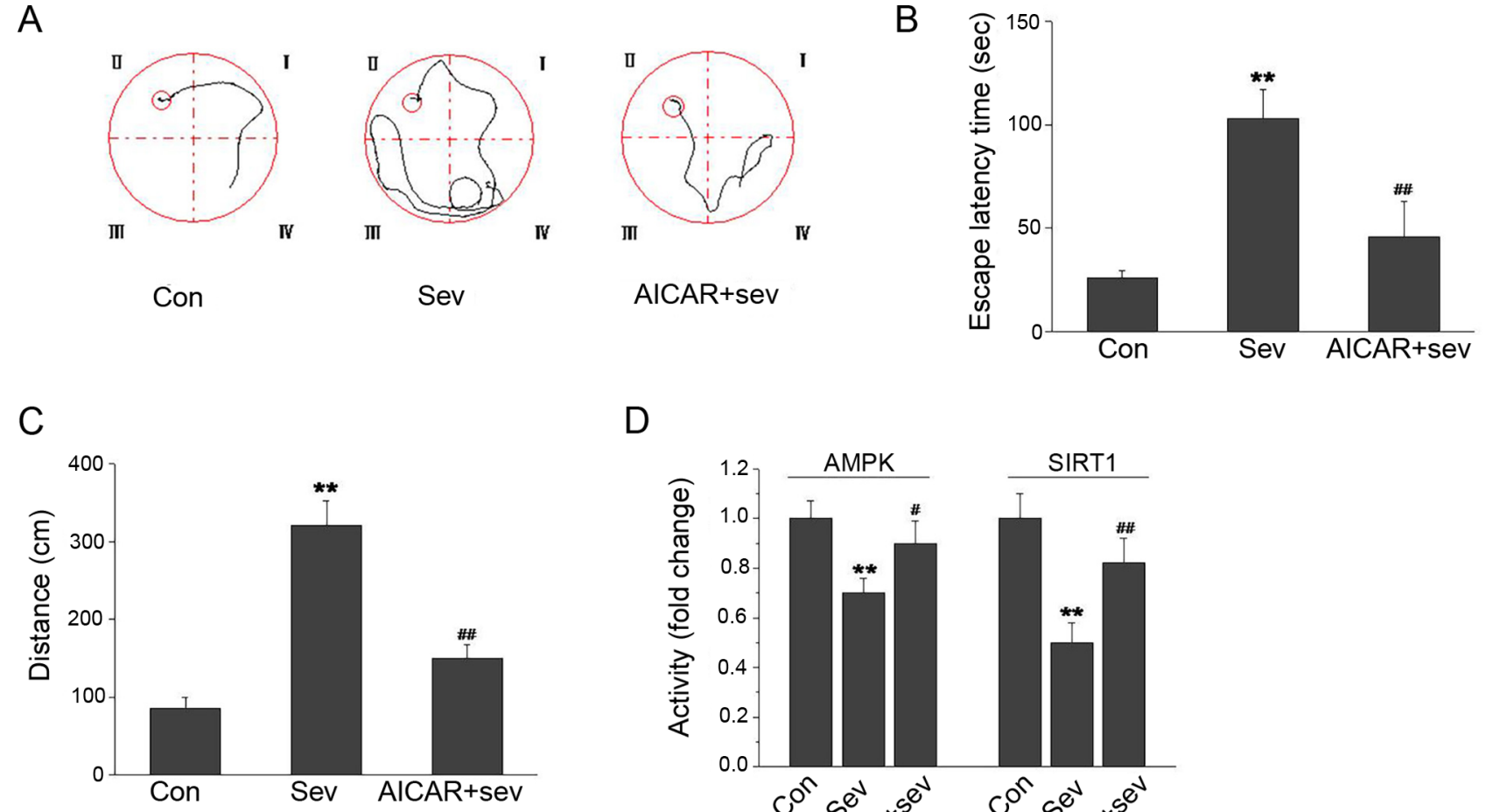

D

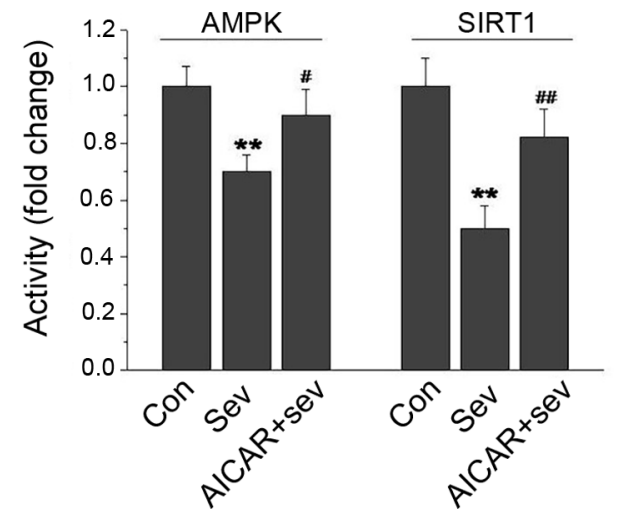

Figure 4. AICAR improves cognition dysfunction and AMPK/SIRT1 activities in Sev-exposedrats. (A) Effects of AICAR on performance in the water Morris maze in Sev-exposedrats. (B) AICAR decreased the escape latency time to the submerged platform in a Morris water maze. (C) AICAR decreased the distance to the submerged platform in the Morris water maze. (D) AICAR increased the levels of AMPK and SIRT1 found in the hippocampus as detected by ELISA. ${ }^{* *} \mathrm{P}<0.01$ vs. Con; ${ }^{\#} \mathrm{P}<0.05,{ }^{\# \#} \mathrm{P}<0.01$ vs. Sev. $\mathrm{n}=8$. Sev, sevoflurane; Con, control; AMPK, AMP-activated protein kinase; SIRT1, Sirtuin 1 .

inhalation induction due to its lack of airway irritation, hemodynamic characteristics and lower pungency (20). Recent studies have reported on hemodynamic and recovery characteristics following anesthesia maintenance with Sev $(21,22)$. Children who have received anesthesia on multiple occasions are more likely to develop cognitive behavioral disorders, while a single anesthetic exposure may have no significant effect on neuro-development (23). A topic of interest is the potential impact of inhalation-based anesthesia in behavioral outcomes such as postoperative cognitive dysfunction and postoperative delirium in pediatric, adult and elderly patients $(22,24,25)$.

Neuronal apoptosis is an important contributor to cognition dysfunction (26). Thus, investigation of the molecular mechanism of Sev-induced neuronal apoptosis may provide a new cognition protection strategy. In past work, numerous genes have been found to be involved in neuronal apoptosis, such as HMGB1/TLR4, caspase3, bcl-2, ROS and P53 (27-29). At present, much study is still needed to elucidate the mechanism of neuronal apoptosis induced by Sev.

AMPK is a major metabolic energy sensor, which may sense energy deficiency in the form of an increased AMP/ATP ratio and regulate metabolic homeostasis through the control of several homeostatic mechanisms, including autophagy and protein degradation (30). AMPK activation has previously been identified as required for SIRT1. SIRT1 is a direct substrate for AMPK phosphorylation (31). The present data has shown that Sev is an effective inducer of neuronal apoptosis. The present study also revealed that inhibition of AMPK/SIRT1 was associated with Sev induced neuronal apoptosis. This was supported with evidence that AMPK activation restrained the neuronal apoptosis induced by Sev. The present study in vivo also revealed that AMPK/SIRT1 inhibition was related to the cognition impairment induced by Sev and that AMPK activation could improve the cognition dysfunction.

Activation of AMPK by phosphorylation of the $\alpha$ subunit at Thr172 helps to maintain the energy balance by switching on a catabolic pathway such as apoptosis induction $(32,33)$. A decreased protein level of p-AMPK/SIRT1 was confirmed to be accompanied by an increased number of Sev induced apoptotic neurons. Furthermore, this effect on apoptosis was blocked by the AMPK activator AICAR, which indicated that AMPK/SIRT1 inhibition was involved in the induction of cognitive impairment by Sev. The protective role of the AMPK activator may be related to the integrity of the brain-blood barrier $(34,35)$. In the animal experiments, rats aged 28 days were selected to simulate human children, as it is desirable to have an experimental basis for pediatric patients with anesthesia in the clinic.

In the present study, AMPK/SIRT1 inhibition was shown to be associated with Sev-induced neuronal apoptosis. AMPK activation restrained the Sev-induced neuronal apoptosis. The present in vivo results also evaluated that AMPK activation was related to cognition dysfunction induced by Sev. In summary, the study revealed the association between the AMPK/SIRT1 pathway, neuronal apoptosis and Sev-induced cognitive dysfunction. These data revealed that AMPK/SIRT1 pathway serves a critical role in Sev-induced neuronal apoptosis. 
AMPK/SIRT1 pathway may be a potential drug target for the therapy of cognitive dysfunction induced by Sev in the future.

\section{Acknowledgements}

Not applicable.

\section{Funding}

The work was financially supported by the China Postdoctoral Science Foundation (grant no. 2015M581308).

\section{Availability of data and materials}

The datasets used and/or analyzed during the current study are available from the corresponding author on reasonable request.

\section{Authors' contributions}

LL designed the study, performed the molecular biology experiments, acquired data and drafted the manuscript. CL established the animal model, performed behavioral testing and molecular biology experiments, collected the behavioral data and revised the manuscript. LF contributed to the conception of the work, performed animal behavioral testing and molecular biology experiments, analyzed and interpreted data, and revised and approved the manuscript. All authors read and approved the final manuscript.

\section{Ethics approval and consent to participate}

Ethics approval was provided by the Animal Experimental Ethical Inspection of Laboratory Animal Center, Shanghai Tenth People's Hospital. This study was performed in strict accordance with the recommendations in the Guide for the Care and Use of Laboratory Animals of the National Institutes of Health.

\section{Patient consent for publication}

Not applicable.

\section{Competing interests}

The authors declare that they have no competing interests.

\section{References}

1. Lim BG, Lee IO, Ahn H, Lee DK, Won YJ, Kim HJ and Kim H: Comparison of the incidence of emergence agitation and emergence times between desflurane and sevoflurane anesthesia in children: A systematic review and meta-analysis. Medicine (Baltimore) 95: e4927, 2016.

2. Sun L: Early childhood general anaesthesia exposure and neurocognitive development. Br J Anaesth 105 (Suppl 1): i61-i8, 2010.

3. Hu X, Wang J, Zhang L, Zhang Q, Duan X and Zhang Y: Postconditioning with sevoflurane ameliorates spatial learning and memory deficit via attenuating endoplasmic reticulum stress induced neuron apoptosis in a rat model of hemorrhage shock and resuscitation. Brain Res 1696: 49-55, 2018.

4. Xu L, Shen J, Yu L, Sun J and Yan M: Autophagy is involved in sevoflurane-induced developmental neurotoxicity in the developing rat brain. Brain Res Bull 140: 226-232, 2018.
5. Voss CM, Pajęcka K, Stridh MH, Nissen JD, Schousboe A and Waagepetersen HS: AMPK activation affects glutamate metabolism in astrocytes. Neurochem Res 40: 2431-2442, 2015.

6. Willows R, Sanders MJ, Xiao B, Patel BR, Martin SR, Read J, Wilson JR, Hubbard J, Gamblin SJ and Carling D: Phosphorylation of AMPK by upstream kinases is required for activity in mammalian cells. Biochem J 474: 3059-3073, 2017.

7. Viollet B, Mounier R, Leclerc J, Yazigi A, Foretz M and Andreelli F: Targeting AMP-activated protein kinase as a novel therapeutic approach for the treatment of metabolic disorders. Diabetes Metab 33: 395-402, 2007.

8. Qian C, Jin J, Chen J, Li J, Yu X, Mo H and Chen G: SIRT1 activation by resveratrol reduces brain edema and neuronal apoptosis in an experimental rat subarachnoid hemorrhage model. Mol Med Rep 16: 9627-9635, 2017.

9. Yan WJ, Wang DB, Ren DQ, Wang LK, Hu ZY, Ma YB, Huang JW and Ding SL: AMPK $\alpha 1$ overexpression improves postoperative cognitive dysfunction in aged rats through AMPK-Sirtl and autophagy signaling. J Cell Biochem: Feb 18, 2019 (Epub ahead of print).

10. Fulco $M$ and Sartorelli V: Comparing and contrasting the roles of AMPK and SIRT1 in metabolic tissues. Cell Cycle 7: 3669-3679, 2008.

11. Salminen A and Kaarniranta K: SIRT1: Regulation of longevity via autophagy. Cell Signal 21: 1356-1360, 2009.

12. Chen Z, Peng IC, Cui X, Li YS, Chien S and Shyy JY: Shear stress, SIRT1, and vascular homeostasis. Proc Natl Acad Sci USA 107: 10268-10273, 2010.

13. Cantó C, Gerhart-Hines Z, Feige JN, Lagouge M, Noriega L, Milne JC, Elliott PJ, Puigserver P and Auwerx J: AMPK regulates energy expenditure by modulating $\mathrm{NAD}^{+}$metabolism and SIRT1 activity. Nature 458: 1056-1060, 2009.

14. An Y, Wang B, Wang X, Dong G, Jia J and Yang Q: SIRT1 Inhibits Chemoresistance and cancer Stemness of gastric cancer by initiating an AMPK/FOXO3 positive feedback loop. Cell Death Dis 11: 115, 2020.

15. Huang J, Wang X, Zhu Y, Li Z, Zhu YT, Wu JC, Qin ZH, Xiang $M$ and Lin F: Exercise activates lysosomal function in the brain through AMPK-SIRT1-TFEB pathway. CNS Neurosci Ther 25: 796-807, 2019.

16. Cetrullo S, D'Adamo S, Tantini B, Borzi RM and Flamigni F: mTOR, AMPK, and Sirt1: Key players in metabolic stress management. Crit Rev Eukaryot Gene Expr 25: 59-75, 2015.

17. Shah SA, Yoon GH, Chung SS, Abid MN, Kim TH, Lee HY and Kim MO: Novel osmotin inhibits SREBP2 via the AdipoR1/AMPK/SIRT1 pathway to improve Alzheimer's disease neuropathological deficits. Mol Psychiatry 22: 407-416, 2017.

18. Wang Q, Li K and Yao S: Effect of inhalational anesthetics on cytotoxicity and intracellular calcium differently in rat pheochromocytoma cells (PC12). J Huazhong Univ Sci Technolog Med Sci 28: 104-109, 2008.

19. Bi C, Cai Q, Shan Y, Yang F, Sun S, Wu X and Liu H: Sevoflurane induces neurotoxicity in the developing rat hippocampus by upregulating connexin 43 via the JNK/c-Jun/AP-1 pathway. Biomed Pharmacother 108: 1469-1476, 2018.

20. Jindal R, Kumra VP, Narani KK and Sood J: Comparison of maintenance and emergence characteristics after desflurane or sevoflurane in outpatient anaesthesia. Indian J Anaesth 55: 36-42, 2011.

21. Choi ES, Shin JY, Oh AY, Park HP, Hwang JW, Lim YJ and Jeon YT: Sevoflurane versus propofol for interventional neuroradiology: A comparison of the maintenance and recovery profiles at comparable depths of anesthesia. Korean J Anesthesiol 66: 290-294, 2014.

22. Parida S and Badhe AS: Comparison of cognitive, ambulatory, and psychomotor recovery profiles after day care anesthesia with propofol and sevofurane. J Anesth 28: 833-838, 2014.

23. Wilder RT, Flick RP, Sprung J, Katusic SK, Barbaresi WJ, Mickelson C, Gleich SJ, Schroeder DR, Weaver AL and Warner DO: Early exposure to anesthesia and learning disabilities in a population-based birth cohort. Anesthesiology 110: 796-804, 2009.

24. Jindal P, Khurana G, Oberoi D and Sharma JP: Recovery profile and emergence delirium following Sevoflurane and Isoflurane anesthesia in children posted for cleft lip surgery. Middle East J Anaesthesiol 21: 679-684, 2012.

25. Chandler JR, Myers D, Mehta D, Whyte E, Groberman MK, Montgomery CJ and Ansermino JM: Emergence delirium in children: A randomized trial to compare total intravenous anesthesia with propofol and remifentanil to inhalational sevofurane anesthesia. Paediatr Anaesth 23: 309-315, 2013. 
26. Yu Y, Feng L, Li J, Lan X, A L, Lv X, Zhang M and Chen L: The alteration of autophagy and apoptosis in the hippocampus of rats with natural aging-dependent cognitive deficits. Behav Brain Res 334: 155-162, 2017.

27. Lu Z, Miao Y, Muhammad I, Tian E, Hu W, Wang J, Wang B, Li R and Li J: Colistin-induced autophagy and apoptosis involves the JNK-Bcl2-Bax signaling pathway and JNK-p53-ROS positive feedback loop in PC-12 cells. Chem Biol Interact 277: 62-73, 2017.

28. Guo X, Shi Y, Du P, Wang J, Han Y, Sun B and Feng J: HMGB1/TLR4 promotes apoptosis and reduces autophagy of hippocampal neurons in diabetes combined with OSA. Life Sci 239: 117020, 2019.

29. Fricker M, Tolkovsky AM, Borutaite V, Coleman M and Brown GC: Neuronal Cell Death. Physiol Rev 98: 813-880, 2018

30. Lau AW, Liu P, Inuzuka H and Gao D: SIRT1 phosphorylation by AMP-activated protein kinase regulates p53 acetylation. Am J Cancer Res 4: 245-255, 2014.

31. Price NL, Gomes AP, Ling AJ, Duarte FV, Martin-Montalvo A, North BJ, Agarwal B, Ye L, Ramadori G, Teodoro JS, et al: SIRT1 is required for AMPK activation and the beneficial effects of resveratrol on mitochondrial function. Cell Metab 15: 675-690, 2012
32. Wang Q, Liu S, Zhai A, Zhang B and Tian G: AMPK-mediated regulation of lipid metabolism by phosphorylation. Biol Pharm Bull 41: 985-993, 2018.

33. Garcia D and Shaw RJ: AMPK: Mechanisms of cellular energy sensing and restoration of metabolic balance. Mol Cell 66: 789-800, 2017.

34. Yu HY, Cai YB and Liu Z: Activation of AMPK improves lipopolysaccharide-induced dysfunction of the blood-brain barrier in mice. Brain Inj 29: 777-784, 2015.

35. Zhao Z, Hu J, Gao X, Liang H and Liu Z: Activation of AMPK attenuates lipopolysaccharide-impaired integrity and function of blood-brain barrier in human brain microvascular endothelial cells. Exp Mol Pathol 97: 386-392, 2014.

(i) This work is licensed under a Creative Commons EY No ND Attribution-NonCommercial-NoDerivatives 4.0 International (CC BY-NC-ND 4.0) License. 